\title{
Another Proof that the Catalan's Constant is Irrational
}

\section{Edigles Guedes ${ }^{1}$ and Prof. Dr. K. Raja Rama Gandhi ${ }^{2}$ \\ Number Theorist, Brazil ${ }^{1}$}

Resource perosn in Mathematics for Oxford University Press and Professor at BITS-Vizag ${ }^{2}$

ABSTRACT. We use the contradiction method for prove, again, that the Catalan's constant is irrational.

\section{INTRODUCTION}

In Mathematics, the Catalan's constant [1] is defined by

$$
G:=\sum_{n=0}^{\infty} \frac{(-1)^{n}}{(2 n+1)^{2}}
$$

The Catalan's constant was named after Eugène Charles February 1894), a French and Belgian mathematician.

In previous paper [2], we prove that the constant $G$ is outra prova de que the constant $G$ is irrational.

\section{THE PROOF}

LEMMA. The Catalan's constant have the following re, ventation in series

$$
G=8 \sum_{n=0}^{\infty} \frac{+1}{(16 \sqrt{2}+10, \sqrt{2})^{2}}
$$

Proof. We developed the power seri s io ula fro the definition of Catalan's constant as follows

$$
\begin{aligned}
& =\frac{(-1)^{0}}{(2 \cdot 0+1)^{2}}, \frac{(-1)}{2 \cdot 1+1)}+\frac{(-1)^{2}}{0 \cdot 2+1)^{2}}+\frac{(-1)^{3}}{(2 \cdot 3+1)^{2}}+\frac{(-1)^{4}}{(2 \cdot 4+1)^{2}}+\frac{(-1)^{5}}{(2 \cdot 5+1)^{2}}+\cdots \\
& =\frac{1}{(2 \cdot 0+1)}+\frac{1}{2 \cdot 2+)^{2}}+\frac{1}{(2 \cdot 4+1)^{2}}+\cdots-\frac{1}{(2 \cdot 1+1)^{2}}-\frac{1}{(2 \cdot 3+1)^{2}}-\frac{1}{(2 \cdot 5+1)^{2}} \cdots \\
& =\frac{1}{2}+\frac{1}{2^{2}}+\frac{1}{(2 \cdot 4+1)^{2}}+\cdots-\left[\frac{1}{(2 \cdot 1+1)^{2}}+\frac{1}{(2 \cdot 3+1)^{2}}+\frac{1}{(2 \cdot 5+1)^{2}}+\cdots\right]
\end{aligned}
$$

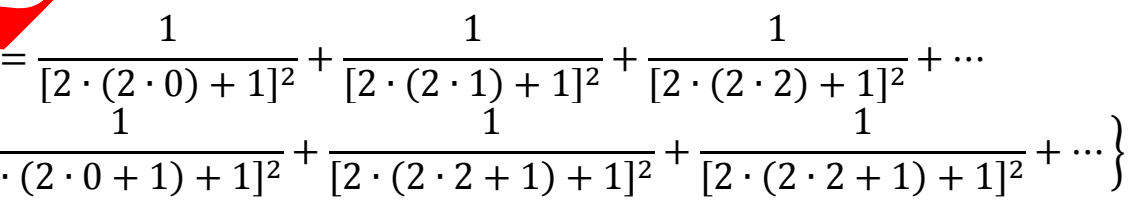

$$
\begin{aligned}
& =\frac{1}{(4 \cdot 0+1)^{2}}+\frac{1}{(4 \cdot 1+1)^{2}}+\frac{1}{(4 \cdot 2+1)^{2}}+\cdots-\left[\frac{1}{(4 \cdot 0+3)^{2}}+\frac{1}{(4 \cdot 1+3)^{2}}+\frac{1}{(4 \cdot 2+3)^{2}}+\cdots\right] \\
& =\sum_{n=0}^{\infty} \frac{1}{(4 n+1)^{2}}-\sum_{n=0}^{\infty} \frac{1}{(4 n+3)^{2}} \\
& =\sum_{n=0}^{\infty} \frac{(4 n+3)^{2}-(4 n+1)^{2}}{(4 n+1)^{2}(4 n+3)^{2}}
\end{aligned}
$$




$$
\begin{aligned}
& =\sum_{n=0}^{\infty} \frac{16 n+8}{(4 n+1)^{2}(4 n+3)^{2}} \\
& =8 \sum_{n=0}^{\infty} \frac{2 n+1}{[(4 n+1)(4 n+3)]^{2}} \\
& =8 \sum_{n=0}^{\infty} \frac{2 n+1}{\left(16 n^{2}+16 n+3\right)^{2}} .
\end{aligned}
$$

THEOREM. The Catalan's constant is irrational.

Proof. We will use the reductio ad absurdum.

By hypothesis, we suppose that $G$ is a rational number. Of course, there ist two po ive integers $a$ and $b$, such that $G=a / b$, where, clearly, $b>1$. Firstly, we define the nu.

$$
\left.x:=\frac{\left(16 b^{2}+16 b+3\right) !^{2}}{4^{8 b^{2}+8 b+1}\left(8 b^{2}+8 b+1\right) !\left(8 b^{2}+8 b\right) !} \cdot\left(G-8 \sum_{n=0}^{b}, \frac{2 n+1}{16}+3\right)^{2}\right)
$$

If $G$ is rational, then $x$ is an integer. We substitute $G=a / b$ nto this a ition to find

$$
\begin{gathered}
x=\frac{\left(16 b^{2}+16 b+3\right) !^{2}}{4^{8 b^{2}+8 b+1}\left(8 b^{2}+8 b+1\right) !\left(8 b^{2}+8 b\right) !} \cdot\left(\frac{a}{b}\right) \\
=\frac{\left(16 b^{2}+16 b+3\right) !^{2} a}{4^{8 b^{2}+8 b+1} b\left(8 b^{2}+8 b+1\right) !\left(8 b^{2}+8 b\right) !}-8 \sum_{n=0}^{b} \frac{\left(16 b^{2}+16 l+3\right) !^{2}(2 n+1)}{4^{8 b^{2}+8 b}} \\
\frac{\left.2 b^{2}+8 b+1\right)}{\left(8 b^{2}+8 b\right) !\left(16 n^{2}+16 n+3\right)^{2}}
\end{gathered}
$$

It is obvious that the first term is an in $r$ becausu, $-0>1$, then $4^{b}(b !)^{2}<(2 b+1) !^{2}$. The second term is an integer; because, for $b>(2 n+1)^{2} 4^{b} b((b-1) !)^{2}<(2 b+1) !^{2}$. Hence $x$ is an integer.

We, now, demonstrate that

First, we demonstrate that the definition of $x$ and we fip

$$
\begin{aligned}
& x=\frac{(2 b+1) !^{2}}{4^{b} b((b-1) !)^{2}} \mid \sum_{n}^{\infty}\left\langle\frac{1}{(2 n+}-\sum_{n}^{b}<_{0} \frac{(-1)^{n}}{(2 n+1)^{2}}\left|=\frac{(2 b+1) !^{2}}{4^{b} b((b-1) !)^{2}}\right| \sum_{n=b+1}^{\infty} \frac{(-1)^{n}}{(2 n+1)^{2}}\right|= \\
& \frac{(2 b+1) !^{2}}{4^{b} b((b-1) !)^{2}}\left|\sum^{\infty}=b+1 \frac{\cos (\pi n)}{+1)^{2}}\right| \geqslant \frac{(2 b+1) !^{2}}{4^{b} b((b-1) !)^{2}}\left|\int_{b+1}^{\infty} \frac{\cos (\pi x)}{(2 x+1)^{2}} d x\right|= \\
& \frac{(2 b+1) !^{2}}{4^{b} b((b-1)}-\frac{-}{4} \quad\left(\left(b, \frac{3}{2}\right) \pi\right)-\frac{\cos (\pi b)}{4 b+6} \mid>0 \text {. }
\end{aligned}
$$

$n$ the other han, for all terms with $2 n+1 \geq 2 b+2$, i.e., $2 n \geq 2 b+1$, we have the upper estimato

$$
\frac{(2 b+1) !}{(2 n+1) !} \leq \frac{1}{(2 b+2)^{2 n-2 b}}
$$

This inequality is strict for every $2 n+1 \geq 2 b+3$, i.e., $n \geq b+1$. Thereof, we substitute (1.1) and (2.4) in (2.1)

$$
\begin{gathered}
x=\frac{(2 b+1) !^{2}}{4^{b} b((b-1) !)^{2}}\left|\sum_{n=0}^{\infty} \frac{(-1)^{n}}{(2 n+1)^{2}}-\sum_{n=0}^{b} \frac{(-1)^{n}}{(2 n+1)^{2}}\right| \\
=\frac{(2 b+1) !^{2}}{4^{b} b((b-1) !)^{2}}\left|\sum_{n=b+1}^{\infty} \frac{(-1)^{n}}{(2 n+1)^{2}}\right|<\frac{(2 b+1) !^{2}}{4^{b} b((b-1) !)^{2}}\left|\sum_{n=b+1}^{\infty} \frac{(-1)^{n}}{(2 n+1) !^{2}}\right|
\end{gathered}
$$




$$
\begin{gathered}
=\frac{1}{4^{b} b((b-1) !)^{2}}\left|\sum_{n=b+1}^{\infty} \frac{(-1)^{n}(2 b+1) !^{2}}{(2 n+1) !^{2}}\right|<\frac{1}{4^{b} b((b-1) !)^{2}}\left|\sum_{n=b+1}^{\infty} \frac{(-1)^{n}}{(2 b+2)^{2 n-2 b}}\right| \\
=\frac{1}{4^{b} b((b-1) !)^{2}}\left|-\frac{(-1)^{b}}{4 b^{2}+8 b+5}\right|<1 .
\end{gathered}
$$

Since there is no integer strictly between 0 and 1 , we have get in a contradiction, and so $G$ must be irrational.

\section{REFERENCES}

[1] http://en.wikipedia.org/wiki/Catalan's_constant, available in July 12, 2013.

[2] Guedes, Edigles, An Elegant Proof that the Catalan's Constant is Irration July 12, . 13, vixra.

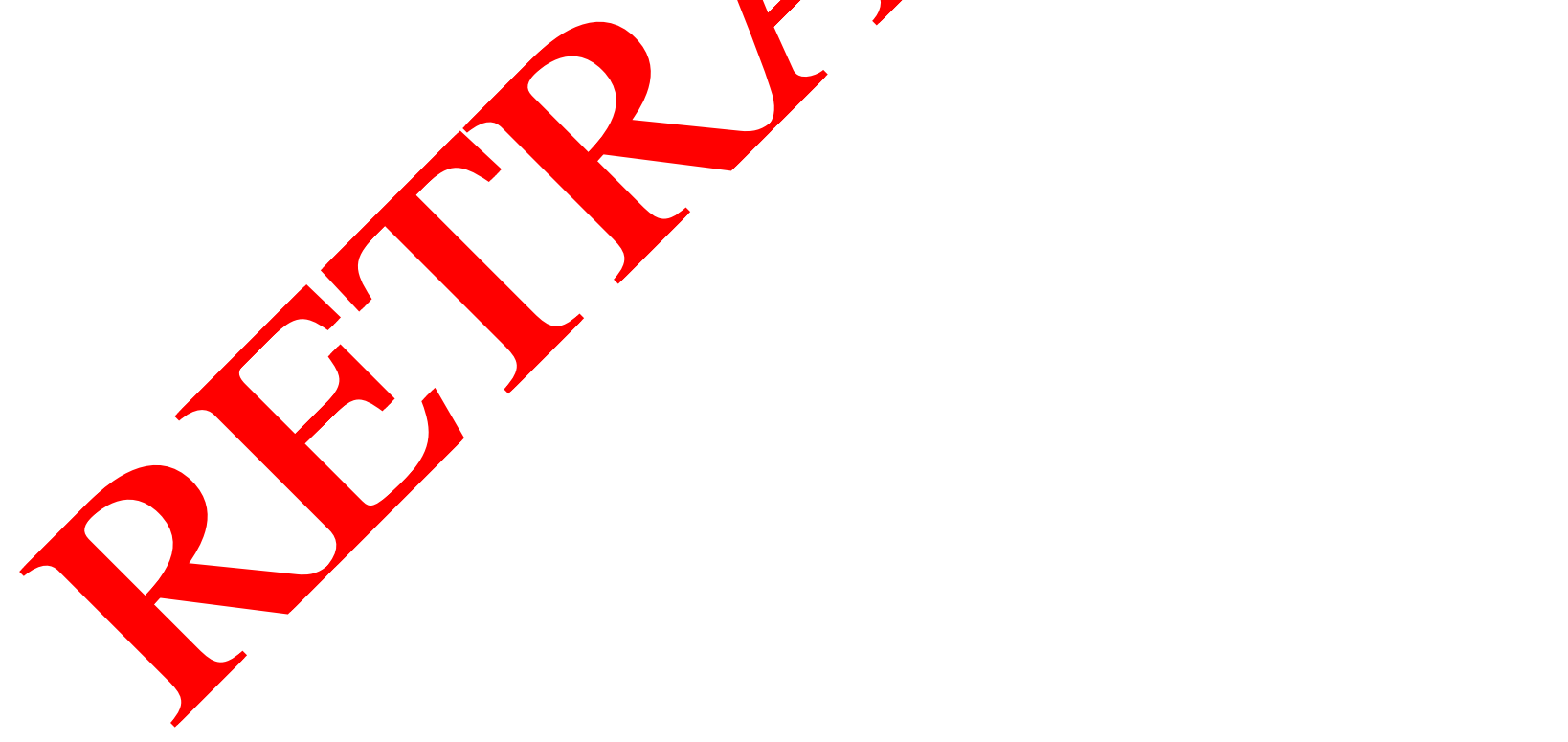

